\title{
EXTREMAL PROBLEMS ON THE CARTAN-HARTOGS DOMAINS
}

\author{
An Wang, Xin Zhao, AND ZhiYin LiU
}

\begin{abstract}
We study some extremal problems on the Cartan-Hartogs domains. Through computing the minimal circumscribed Hermitian ellipsoid of the Cartan-Hartogs domains, we get the Carathéodory extremal mappings between the Cartan-Hartogs domains and the unit hyperball, and the explicit formulas for computing the Carathéodory extremal value.
\end{abstract}

\section{Part I. Introduction}

In the theory of single complex variable, Riemann mapping theorem has the important theoretical and practical meaning. According to the theorem, the problem about the classification of simple connected domains in the complex plane has been resolved. To find the extremal function of the extremal problem

$$
\sup _{f \in \widetilde{F}(\Omega)}\left|f^{\prime}\left(z_{0}\right)\right|\left(z_{0} \in \Omega\right)
$$

is a key step of proving the Riemann mapping theorem, where $\Omega$ is a simply connected domain in $\mathbb{C}$ with at least two boundary points and $\widetilde{F}(\Omega)$ is the family of all functions $f$ such that $f$ maps $\Omega$ conformally onto the unit disk, it is holomorphic and $|f(z)|<1$ in $\Omega$.

As is well known, Riemann mapping theorem fails for domains in $\mathbb{C}^{n}$ with $n>1$. But the similar extremal problem is still significant to study in the theory of several complex variables: Let $\mathcal{M}$ be a domain in $\mathbb{C}^{n}$ and $q \in \mathcal{M}$. Let $\mathcal{M}_{q}$ denote the couple $(\mathcal{M}, q)$, a "pointed domain". For two pointed domains $\mathcal{M}_{q_{1}}$ and $\mathcal{N}_{q_{2}}$, let $\operatorname{Hol}\left(\mathcal{M}_{q_{1}}, \mathcal{N}_{q_{2}}\right)$ denote the set of holomorphic maps from $\mathcal{M}$ to $\mathcal{N}$ that send $q_{1}$ to $q_{2}$. A map $f \in \operatorname{Hol}\left(\mathcal{M}_{q_{1}}, \mathcal{N}_{q_{2}}\right)$ is said to be Carathéodory extremal map, if

$$
\left|\operatorname{det} d f\left(q_{1}\right)\right|=\sup \left\{\left|\operatorname{det} d g\left(q_{1}\right)\right|: g \in \operatorname{Hol}\left(\mathcal{M}_{q_{1}}, \mathcal{N}_{q_{2}}\right)\right\},
$$

Received January 14, 2006.

2000 Mathematics Subject Classification. 32H02, 32F 45.

Key words and phrases. extremal problem, Cartan-Hartogs domain, minimal circumscribed Hermitian ellipsoid.

Research supported by NSF of China (Grant No.10471097), and Scientific Research Common Program of Beijing Municipal Commission of Education (Grant KM200410028002). 
where $d g\left(q_{1}\right)$ denotes the Jacobian matrix of $g$ at the point of $q_{1}$, and now $\left|\operatorname{det} d f\left(q_{1}\right)\right|$ is called to be Carathéodory extremal value. Simply, we call them C-extremal mapping and C-extremal value. The classical problem can be considered as an extension of the classical Schwarz lemma in high dimensions [3].

For the extremal problem, the important part is the computing problem for extremal mapping and extremal value in explicit formulas. Carathéodory firstly studied the C-extremal mapping and obtained the explicit formula for C-extremal mapping from the polydisc into the unit hyperball $B^{n}$ in 1932 [1]. Kubota obtained the explicit formulas for C-extremal mappings and values from the Cartan domains into the unit hyperball $B^{n}$ with the method of series expansion [4], furthermore he discussed the extremal problems between all the bounded symmetric domains and $B^{n}$ [5]. He proved that the extremal mappings which he obtained are unique up to unitary linear transformations. Ma Daowei obtained the explicit formulas for the C-extremal mappings from convex generalized ellipsoid to $B^{n}$, and gave the formulas for the C-extremal mappings from $B^{n}$ to generalized ellipsoid, which may not be convex [3].

In terms of C-extremal mappings, the extremal distance between two pointed domains is defined to be

$$
\mu\left(\mathcal{M}_{q_{1}}, \mathcal{N}_{q_{2}}\right)=-\log \left|\operatorname{det} d(g \circ f)\left(q_{1}\right)\right|,
$$

where $\mathcal{M}, \mathcal{N}$ are two bounded domains in $\mathbb{C}^{n}$ and $q_{1} \in \mathcal{M}, q_{2} \in \mathcal{N}$, moreover $f \in \operatorname{Hol}\left(\mathcal{M}_{q_{1}}, \mathcal{N}_{q_{2}}\right), g \in \operatorname{Hol}\left(\mathcal{N}_{q_{2}}, \mathcal{M}_{q_{1}}\right)$ are C-extremal mappings.

Ma Daowei also obtained the extremal distance between the complex ellipsoid and $B^{n}[3]$, and the extremal distance between the strongly pseudoconvex domain and $B^{n}[2]$. The extremal distance is an effective means for estimating Kobayashi metric, Carathéodory metric, Sibony metric and Eisenman volume forms of domains in $\mathbb{C}^{n}$.

Yin Weiping and G. Roos constructed four types of domains called CartanHartogs domains [7], and the four types of Cartan-Hartogs domains are:

$$
\begin{gathered}
Y_{I}(N ; m, n ; K):=\left\{W \in \mathbf{C}^{N}, Z \in \Re_{I}(m, n):\|W\|^{2 K}<\operatorname{det}\left(I-Z \bar{Z}^{t}\right), K>0\right\}, \\
Y_{I I}(N, p, K):=\left\{W \in \mathbf{C}^{N}, Z \in \Re_{I I}(p):\|W\|^{2 K}<\operatorname{det}\left(I-Z \bar{Z}^{t}\right), K>0\right\}, \\
Y_{I I I}(N, q, K):=\left\{W \in \mathbf{C}^{N}, Z \in \Re_{I I I}(q):\|W\|^{2 K}<\operatorname{det}\left(I-Z \bar{Z}^{t}\right), K>0\right\}, \\
Y_{I V}(N, n, K):=\left\{W \in \mathbf{C}^{N}, Z \in \Re_{I V}(n):\|W\|^{2 K}<1-2 z \bar{z}^{t}+\left|z z^{t}\right|^{2}, K>0\right\},
\end{gathered}
$$
where $\Re_{\alpha}, \alpha=I, I I, I I I, I V$ denote the four types of Cartan domain in the sense of L. K. Hua, $Z^{t}$ denotes the transposed of $Z$, det denotes the determinant of a square matrix, $N$ is a positive integer, and $K$ is a positive real number.

In this paper, we discuss the Carathéodory extremal problems on the CartanHartogs domains. The form of $Y_{I I}(N, p, K)$ is more special, the method is skillful and have a difficulty to get the minimal circumscribed Hermitian ellipsoid of $Y_{I I}(N, p, K)$, so we prove the conclusions about the C-extremal mappings and C-extremal values of $Y_{I I}(N, p, K)$ in detail. But we can use the similar method to get the C-extremal mappings and C-extremal values on $Y_{I}(N ; m, n ; K)$ and 
$Y_{I I I}(N, q, K)$, so we list the main results about the Carathéodory extremal problems on the two Cartan-Hartogs domains in the last part of the paper.

\section{Part II. Preliminaries}

For two pointed domains $\mathcal{M}_{q_{1}}, \mathcal{N}_{q_{2}} \subset \mathbb{C}^{n}$, let

$$
J_{\max }\left(\mathcal{M}_{q_{1}}, \mathcal{N}_{q_{2}}\right)=\sup \left\{\left|\operatorname{det} d g\left(q_{1}\right)\right|: g \in \operatorname{Hol}\left(\mathcal{M}_{q_{1}}, \mathcal{N}_{q_{2}}\right)\right\} .
$$

Obviously,

$$
\mu\left(\mathcal{M}_{q_{1}}, \mathcal{N}_{q_{2}}\right)=-\log \left[J_{\max }\left(\mathcal{M}_{q_{1}}, \mathcal{N}_{q_{2}}\right) \cdot J_{\max }\left(\mathcal{N}_{q_{2}}, \mathcal{M}_{q_{1}}\right)\right] .
$$

If $\mathcal{M}$ and $\mathcal{N}$ contain origins, we write

$$
J_{\max }(\mathcal{M}, \mathcal{N}):=J_{\max }\left(\mathcal{M}_{0}, \mathcal{N}_{0}\right), \quad \mu(\mathcal{M}, \mathcal{N})=\mu\left(\mathcal{M}_{0}, \mathcal{N}_{0}\right)
$$

Proposition 1 ([3]). If $D_{1}, D_{2}$ are balanced domains (i.e., $c z \in D_{i}$ for $c \in$ $\mathbb{C},|c| \leqslant 1$ and $z \in D_{i}(i=1,2)$ ) and if $D_{2}$ is a holomorphic domain, then any holomorphic mapping $f \in \operatorname{Hol}\left(\left(D_{1}, 0\right),\left(D_{2}, 0\right)\right)$ satisfies $d f(0)\left(D_{1}\right) \subset D_{2}$.

Proof. See [3].

By Proposition 1,

$$
J_{\max }\left(D_{1}, D_{2}\right)=\sup \left\{|\operatorname{det} l|: l \text { complex linear map, } l\left(D_{1}\right) \subset D_{2}\right\} .
$$

Definition 2 ([3]). An Hermitian ellipsoid centered at origin is a domain of the form:

$$
\left\{z \in \mathbb{C}^{n}: z A \bar{z}^{t}<1\right\}
$$

or

$$
\left\{z \in \mathbb{C}^{n}: \sum_{j, k=1}^{n} a_{j k} z_{j} \bar{z}_{k}<1\right\},
$$

where $A=\left(a_{j k}\right)$ is a positive definite Hermitian matrix.

Proposition 3. For a Hermitian ellipsoid $S=\left\{z \in \mathbb{C}^{n}: \sum_{j, k=1}^{n} a_{j k} z_{j} \bar{z}_{k}<1\right\}$, $V(S)=\frac{1}{\left|\operatorname{det}\left(a_{j k}\right)\right|} \omega_{n}$, where $V(S)$ denotes the volume of $S$, and $\omega_{n}$ denotes the volume of the unit hyperball of dimension $n$.

Proof. As $\left(a_{j k}\right)$ is a positive definite Hermitian matrix of order $n$, there exists a unitary matrix $U$, such that

$$
\left(a_{j k}\right)=\bar{U}^{t} \Lambda^{2} U
$$

where

$$
\Lambda=\left(\begin{array}{cccc}
\sqrt{\lambda_{1}} & 0 & \cdots & 0 \\
0 & \sqrt{\lambda_{2}} & \cdots & 0 \\
\cdots & \cdots & \cdots & \cdots \\
0 & 0 & \cdots & \sqrt{\lambda_{n}}
\end{array}\right) \quad \lambda_{1} \geqslant \lambda_{2} \geqslant \cdots \geqslant \lambda_{n}>0
$$


Then

$$
\left(z_{1}, \ldots, z_{n}\right)\left(a_{j k}\right)\left(\bar{z}_{1}, \ldots, \bar{z}_{n}\right)^{t}=\left(z_{1}, \ldots, z_{n}\right) \bar{U}^{t} \Lambda^{2} U\left(\bar{z}_{1}, \ldots, \bar{z}_{n}\right)^{t}
$$

Hence we get a linear transformation

$$
\begin{gathered}
l: S \longrightarrow B^{n} \\
\left(z_{1}, \ldots, z_{n}\right) \mapsto\left(z_{1}, \ldots, z_{n}\right) \bar{U}^{t} \Lambda .
\end{gathered}
$$

Let $\omega_{n}$ be the volume of $B^{n}$, then

$$
V(S)=\int_{S} d_{V(S)}=\int_{B^{n}} \frac{1}{|\operatorname{det} d l|^{2}} d_{\omega_{n}}=\frac{1}{\left|\operatorname{det}\left(a_{j k}\right)\right|} \omega_{n}
$$

Proposition 4 ([3]). Let $D$ be a domain in $\mathbb{C}^{n}$, containing the origin. If $l$ is a complex linear map such that $l(D) \subset B^{n}$, then $l^{-1}\left(B^{n}\right)$ is a Hermitian ellipsoid containing $D$. If $l$ is a solution of the extremal problem

$$
\sup \left\{|\operatorname{det} l|: l \text { complex linear map, } l(D) \subset B^{n}\right\},
$$

then $l^{-1}\left(B^{n}\right)$ is a circumscribed Hermitian ellipsoid of $D$ of least volume, or a minimal circumscribed Hermitian ellipsoid. If $m$ is a solution of the extremal problem

$$
\sup \left\{|\operatorname{det} m|: m \text { complex linear map, } m\left(B^{n}\right) \subset D\right\},
$$

then $m\left(B^{n}\right)$ is an inscribed Hermitian ellipsoid of $D$ of greatest volume, or a maximal inscribed Hermitian ellipsoid.

Proof. See [3].

Let $D$ be a bounded domain in $\mathbb{C}^{n}$, we write $Q(D)$ to be the minimal circumscribed Hermitian ellipsoid of $D$, and $P(D)$ to be the maximal inscribed Hermitian ellipsoid of $D$.

Proposition 5. Let $D_{1}$ and $D_{2}$ be bounded domains, if $l \in G L(n, \mathbb{C})$, and $l\left(D_{1}\right)=D_{2}$, then $l\left(Q\left(D_{1}\right)\right)=Q\left(D_{2}\right)$ and $l\left(P\left(D_{1}\right)\right)=P\left(D_{2}\right)$.

$G L(n, \mathbb{C})$ denotes the set which consists of nonsingular matrix of order $n$ in $\mathbb{C}$, and it is called the general linear group in $\mathbb{C}$.

Proof. Let $L$ be a solution of the following extremal problem

$$
\sup \left\{|\operatorname{det} \rho|: \rho \text { complex linear map, } \rho\left(D_{1}\right) \subset B^{n}\right\} \text {. }
$$

By Proposition $4, L^{-1}\left(B^{n}\right)$ is the minimal circumscribed ellipsoid of $D_{1}$, and it is written to be $Q\left(D_{1}\right)$.

Since $l \in G L(n, \mathbb{C})$ and $l\left(D_{1}\right)=D_{2}$, it follows that $L \circ l^{-1}$ is a solution of the extremal problem

$$
\sup \left\{\left|\operatorname{det} \rho^{\prime}\right|: \rho^{\prime} \text { complex linear map, } \rho^{\prime}\left(D_{2}\right) \subset B^{n}\right\},
$$

furthermore

$$
\left(L \circ l^{-1}\right)^{-1}\left(B^{n}\right)=l\left(L^{-1}\left(B^{n}\right)\right)=l\left(Q\left(D_{1}\right)\right)=Q\left(D_{2}\right) .
$$


By the same means, we can get $l\left(P\left(D_{1}\right)\right)=P\left(D_{2}\right)$.

Proposition 6. Let $D$ be a bounded domain in $\mathbb{C}^{n}$. Then $D$ has minimal circumscribed and maximal inscribed Hermitian ellipsoid, and the minimal circumscribed Hermitian ellipsoid of $D$ is unique. In addition, if $D$ is convex and balanced, then the maximal inscribed Hermitian ellipsoid is also unique.

Proof. See [3].

For the convenience of the following discussion, we let

$$
M=\frac{p(p+1)}{2} .
$$

Proposition 7. Both $B^{N+M}$ and $Y_{I I}(N, p, K)$ are balanced domains.

Proof. Obviously, $B^{N+M}$ is a balanced domain.

Next we prove that $Y_{I I}(N ; p ; K)$ is a balanced domain: If

$$
\forall(w, Z) \in Y_{I I}(N ; p ; K)
$$

and $\forall c \in \mathbb{C},|c| \leqslant 1$, then $c Z \in \Re_{I I}(p)$, and $\|c w\|^{2 K}<\operatorname{det}(I-c Z \bar{c} \bar{Z})$.

For $Z \in \Re_{I I}(p)$, there exists a unitary matrix $U^{[4]}$ such that

$$
Z=U^{t} \tilde{\Lambda} U
$$

and

$$
I-Z \bar{Z}=U^{t}\left(I-\widetilde{\Lambda}^{2}\right) \bar{U}>0
$$

where

$$
\widetilde{\Lambda}=\left(\begin{array}{cccc}
\tilde{\lambda}_{1} & 0 & \cdots & 0 \\
0 & \tilde{\lambda}_{2} & \cdots & 0 \\
\cdots & \cdots & \cdots & \cdots \\
0 & 0 & \cdots & \tilde{\lambda}_{p}
\end{array}\right) \quad \tilde{\lambda}_{1} \geqslant \tilde{\lambda}_{2} \geqslant \cdots \geqslant \tilde{\lambda}_{p} \geqslant 0
$$

then $0 \leqslant \lambda_{i}^{2}<1, i=1, \ldots, p$.

Here

$$
I-c Z \bar{c} \bar{Z}=I-|c|^{2} Z \bar{Z}=U^{t}\left(I-|c|^{2} \tilde{\Lambda}^{2}\right) \bar{U} .
$$

Because $|c|^{2} \leqslant 1,1-|c|^{2} \lambda_{i}^{2} \geqslant 1-\lambda_{i}^{2}>0, i=1, \ldots, p$.

Thus $I-c Z \bar{c} \bar{Z}>0$ and $c Z=(c Z)^{t}$, i.e., $c Z \in \Re_{I I}(p)$.

Furthermore

$$
\operatorname{det}(I-Z \bar{Z}) \leqslant \operatorname{det}(I-c Z \bar{c} \bar{Z})
$$

so

$$
\|c w\|^{2 K} \leqslant\|w\|^{2 K}<\operatorname{det}(I-Z \bar{Z}) \leqslant \operatorname{det}(I-c Z \bar{c} \bar{Z}) .
$$

Hence the proposition is true.

In the same way, we can get that both $Y_{I}(N ; m, n ; k)$ and $Y_{I I I}(N, q, K)$ are balanced domains. 


\section{Part III. Extremal problem on $Y_{I I}(N, p, k)$}

\section{The form of the minimal circumscribed Hermitian ellipsoid}

Lemma 8 ([8]). Let $A_{n \times n}$ and $B_{n \times n}$ be two positive definite Hermitian matrices. If

then $A=B$.

$$
\left\{z \in \mathbb{C}^{n}: z A \bar{z}^{t}<1\right\}=\left\{z \in \mathbb{C}^{n}: z B \bar{z}^{t}<1\right\},
$$

Proof. See [8].

Explanation [6]: In order to do the following discussion, we let

$$
Z=\left(\frac{z_{j k}}{\sqrt{2} p_{j k}}\right)_{1 \leqslant j, k \leqslant p}
$$

be symmetrical matrix of order $p$, where

$$
p_{j k}=\left\{\begin{array}{cc}
\frac{1}{\sqrt{2}}, & j=k \\
1, & j \neq k
\end{array}\right.
$$

We arrange the elements of the matrix $Z$ in the form of a vector in $\mathbb{C}^{M}$ according to the following sequence:

$$
z=\left(z_{11}, z_{12}, \ldots, z_{1 p}, z_{22}, \ldots, z_{2 p}, \ldots, z_{p p}\right),
$$

and $\|z\|^{2}=\operatorname{tr}\left(Z \bar{Z}^{t}\right)$.

Proposition 9. The minimal circumscribed Hermitian ellipsoid of $Y_{I I}(N, p, K)$ must have the form:

$$
S(a, b)=\left\{(w, z) \in \mathbb{C}^{N+M}: a\|w\|^{2}+b\|z\|^{2}<1\right\} \quad a>0, b>0 .
$$

Proof. For $\forall(w, Z) \in Y_{I I}(N, p, K)$, we write $w=\left(w_{1}, w_{2}, \ldots, w_{N}\right)$, and $Z=$ $\left(\frac{z_{j k}}{\sqrt{2} p_{j k}}\right)_{1 \leqslant j, k \leqslant p} \in \Re_{I I}(p)$, where $p_{j k}$ is the same as the definition in the explanation.

Now we think about the following mappings:

$$
\begin{aligned}
& \text { 1. } \xi_{\gamma}: Y_{I I}(N, p, K) \quad \longrightarrow \quad Y_{I I}(N, p, K) \quad \gamma=1, \ldots, N \text {. } \\
& w_{\gamma} \mapsto-w_{\gamma} \\
& w_{\delta} \mapsto w_{\delta} \quad \delta=1, \ldots, \gamma-1, \gamma+1, \ldots, N . \\
& z_{j k} \mapsto z_{j k} \quad j=1, \ldots, p ; k=1, \ldots, p ; j \leqslant k .
\end{aligned}
$$

2. $\xi_{u v}: Y_{I I}(N, p, K) \rightarrow Y_{I I}(N, p, K) \quad 1 \leqslant u<v \leqslant N$.

$$
\begin{array}{rlll}
w_{u} & \mapsto w_{v} & \\
w_{v} & \mapsto w_{u} & \\
w_{\delta} & \mapsto w_{\delta} & \delta=1, \ldots, N, \text { and } \delta \neq u, v . \\
z_{j k} & \mapsto z_{j k} & j=1, \ldots, p ; k=1, \ldots, p ; j \leqslant k . \\
K) & \mapsto Y_{I I}(N, p, K) & r=1, \ldots, p . \\
w_{\delta} & \mapsto w_{\delta} & \delta=1, \ldots, N . \\
z & \mapsto \quad z\left[A_{r} \cdot \times A_{r}\right]_{s}^{t} &
\end{array}
$$

3. $\eta_{r}: Y_{I I}(N, p, K) \rightarrow Y_{I I}(N, p, K) \quad r=1, \ldots, p$. 
$A_{r}=I-2 I_{r r}$, where $I$ is a unit matrix of order $p$, and $I_{m n}$ is a matrix of order $p$, in whose elements the element on the crossed-point of line $m$ and column $n$ is 1 and others are 0 .

$[A \times A]_{s}$ is the symmetric Kronecker product of two square matrices $A$ [6]. Let $A=\left(\theta_{c d}\right)_{p \times p}$, and the indexing sets $(c \tau)(c \leqslant \tau),(d \omega)(d \leqslant \omega)$ are ranged with the fixed sequence $(1,1),(1,2), \ldots,(1, p),(2,2), \ldots,(2, p), \ldots,(p, p)$, then the element $\theta_{(c \tau)(d \omega)}$ on the crossed-point of line $(c \tau)$ and column $(d \omega)$ of $[A \times A]_{s}$ is

where

$$
\theta_{(c \tau)(d \omega)}=p_{c \tau} p_{d \omega}\left(\theta_{c d} \theta_{\tau \omega}+\theta_{c \omega} \theta_{\tau d}\right), c \leqslant \tau, d \leqslant \omega,
$$

$$
p_{c \tau}=\left\{\begin{array}{cc}
\frac{1}{\sqrt{2}}, & c=\tau, \\
1, & c \neq \tau .
\end{array}\right.
$$

4. $\eta_{\alpha \beta}: \quad Y_{I I}(N, p, K) \quad \longrightarrow \quad Y_{I I}(N, p, K) \quad 1 \leqslant \alpha<\beta \leqslant p$.

$$
\begin{array}{rlr}
w_{\delta} & \mapsto w_{\delta} & \delta=1, \ldots, N . \\
z & \mapsto z\left[A_{\alpha \beta} \cdot \times A_{\alpha \beta}\right]_{s}^{t} &
\end{array}
$$

where $A_{\alpha \beta}=I-I_{\alpha \alpha}-I_{\beta \beta}+I_{\alpha \beta}+I_{\beta \alpha}$.

Obviously, these mappings such as $\xi_{\gamma}, \xi_{u v}, \eta_{r}, \eta_{\alpha \beta}$ are in $G L(N+M, \mathbb{C})$, and $\xi_{\gamma}\left(Y_{I I}\right)=Y_{I I}, \xi_{u v}\left(Y_{I I}\right)=Y_{I I}, \eta_{r}\left(Y_{I I}\right)=Y_{I I}, \eta_{\alpha \beta}\left(Y_{I I}\right)=Y_{I I}$.

Let $S(a, b)$ be the minimal circumscribed Hermitian ellipsoid of $Y_{I I}(N, p, K)$, according to Proposition $5, \xi_{\gamma}(S(a, b))=S(a, b), \xi_{u v}(S(a, b))=S(a, b)$, $\eta_{r}(S(a, b))=S(a, b), \eta_{\alpha \beta}(S(a, b))=S(a, b)$.

Let the form of $S(a, b)$ be

$$
S(a, b)=\left\{(w, z) \in \mathbb{C}^{N+M}:(w, z)\left(\begin{array}{cc}
A & C \\
\bar{C}^{t} & B
\end{array}\right)(\bar{w}, \bar{z})^{t}<1\right\},
$$

where

$$
\left(\begin{array}{cc}
A & C \\
\bar{C}^{t} & B
\end{array}\right)
$$

is a positive definite Hermitian matrix, $A$ is a square matrix of order $N$, and $B$ is a square matrix of order $M$.

Because $\xi_{\gamma}(S(a, b))=S(a, b), \gamma=1, \ldots, N$, we know from Lemma 8

and $C=0$.

$$
A=\left(\begin{array}{cccc}
a_{1} & 0 & \cdots & 0 \\
0 & a_{2} & \cdots & 0 \\
\cdots & \cdots & \cdots & \cdots \\
0 & 0 & \cdots & a_{N}
\end{array}\right)
$$

Because $\xi_{u v}(S(a, b))=S(a, b), 1 \leqslant u<v \leqslant p$, we know by Lemma 8

$$
A=\left(\begin{array}{cccc}
a & 0 & \cdots & 0 \\
0 & a & \cdots & 0 \\
\cdots & \cdots & \cdots & \cdots \\
0 & 0 & \cdots & a
\end{array}\right),
$$

where $a>0$. 
For $\eta_{r}(S(a, b))=S(a, b)$, according to Lemma 8

$$
\left(\begin{array}{cc}
I^{(N)} & 0 \\
0 & {\left[A_{r} \cdot \times A_{r}\right]_{s}^{t}}
\end{array}\right)\left(\begin{array}{cc}
A & 0 \\
0 & B
\end{array}\right)\left(\begin{array}{cc}
I^{(N)} & 0 \\
0 & {\left[A_{r} \cdot \times A_{r}\right]_{s}^{t}}
\end{array}\right)=\left(\begin{array}{cc}
A & 0 \\
0 & B
\end{array}\right) .
$$

With the inductive approach, we obtain

$$
B=\left(\begin{array}{cccc}
b_{1} & 0 & \cdots & 0 \\
0 & b_{2} & \cdots & 0 \\
\cdots & \cdots & \cdots & \cdots \\
0 & 0 & \cdots & b_{M}
\end{array}\right)
$$

By the transformation $\eta_{\alpha \beta}$ and Lemma 8, we can get

$$
B=\left(\begin{array}{cccc}
b & 0 & \cdots & 0 \\
0 & b & \cdots & 0 \\
\cdots & \cdots & \cdots & \cdots \\
0 & 0 & \cdots & b
\end{array}\right), b>0
$$

Generalizing from the above proof, the form of the minimal circumscribed Hermitian ellipsoid of $Y_{I I}(N, p, K)$ is

$$
S(a, b)=\left\{(w, z) \in \mathbb{C}^{N+M}: a\|w\|^{2}+b\|z\|^{2}<1\right\} \quad a>0, b>0 .
$$

In the same way, we get that the form of the maximal inscribed Hermitian ellipsoid of $Y_{I I}(N, p, K)$ is

$$
R(c, d)=\left\{(w, z) \in \mathbb{C}^{N+M}: c\|w\|^{2}+d\|z\|^{2}<1\right\} \quad c>0, d>0 .
$$

\section{The inscribed ball of $Y_{I I}(N, p, K)$}

Lemma 10 ([8]). If there are $a_{1}, a_{2}, \ldots, a_{n} \geqslant-1$, and the nonzero numbers among them are all positive or negative, then $\left(1+a_{1}\right)\left(1+a_{2}\right) \cdots\left(1+a_{n}\right) \geqslant$ $1+\left(a_{1}+a_{2}+\cdots+a_{n}\right)$.

Proof. See [8].

Proposition 11. When $K \geqslant 1$, the unit hyperball $B^{N+M}$ is the maximal inscribed ball of $Y_{I I}(N, p, K)$.

Proof. Firstly, we prove $B^{N+M} \subset Y_{I I}(N, p, K)$.

Because $B^{N+M}$ and $Y_{I I}(N, p, K)$ are all the balanced domains, we only need to prove $I-Z \bar{Z} \geqslant 0$, and $\|w\|^{2 K} \leqslant \operatorname{det}(I-Z \bar{Z})$, when $\forall(w, Z) \in \partial B^{N+M}$ i.e., $\|w\|^{2}+\operatorname{tr}(Z \bar{Z})=1$.

For the symmetric matrix $Z$ of order $p$, there exists a unitary matrix $U$, such that

$$
Z=U^{t} \widetilde{\Lambda} U
$$

where $\widetilde{\Lambda}$ is the same as the equation $(\star)$, then

$$
\operatorname{tr}(Z \bar{Z})=\operatorname{tr}\left(U^{t} \widetilde{\Lambda}^{2} \bar{U}\right)=\operatorname{tr}\left(\bar{U} U^{t} \widetilde{\Lambda}^{2}\right)
$$




$$
=\tilde{\lambda}_{1}^{2}+\tilde{\lambda}_{2}^{2}+\cdots+\tilde{\lambda}_{p}^{2} .
$$

Because $\|w\|^{2}+\operatorname{tr}(Z \bar{Z})=1$, we know $\operatorname{tr}(Z \bar{Z}) \leqslant 1$, furthermore $1 \geqslant \tilde{\lambda}_{1}^{2} \geqslant$ $\tilde{\lambda}_{2}^{2} \geqslant \cdots \geqslant \tilde{\lambda}_{p}^{2} \geqslant 0$, and $I-Z \bar{Z} \geqslant 0$.

Because $\|w\|^{2}=1-\left(\tilde{\lambda}_{1}^{2}+\cdots+\tilde{\lambda}_{p}^{2}\right)$, when $K \geqslant 1$, by Lemma 10 , we get:

$$
\|w\|^{2 K} \leqslant\|w\|^{2}=1-\left(\tilde{\lambda}_{1}^{2}+\cdots+\tilde{\lambda}_{p}^{2}\right) \leqslant\left(1-\tilde{\lambda}_{1}^{2}\right) \cdots\left(1-\tilde{\lambda}_{p}^{2}\right)=\operatorname{det}(I-Z \bar{Z}) .
$$

In summary, $B^{N+M} \subset Y_{I I}(N, p, K)$.

Secondly, we prove that there are common points on the boundary of $B^{N+M}$ and $Y_{I I}(N, p, K)$.

Let

$$
e_{i}=(\underbrace{0, \ldots, 0}_{i-1}, 1,0, \ldots, 0)^{t} \in \mathbb{C}^{N+M}, i=1,2, \ldots, N,
$$

then $e_{i} \in \partial B^{N+M}$, and it is easy to check that $e_{i} \in \partial Y_{I I}(N, p, K)$. So $e_{i}(i=$ $1,2, \ldots, N)$ are the tangent points of $B^{N+M}$ and $Y_{I I}(N, p, K)$.

At last, we prove that $B^{N+M}$ is the unique maximal inscribed ball of $Y_{I I}(N, p, K)$.

we have known that the form of the maximal inscribed Hermitian ellipsoid of $Y_{I I}(N, p, K)$ is

$$
R(c, d)=\left\{(w, z) \in \mathbb{C}^{N+M}: c\|w\|^{2}+d\|z\|^{2}<1\right\} \quad c>0, d>0 .
$$

For $\forall(w, Z) \in \partial Y_{I I}(N, p, K)$, there is $c\|w\|^{2}+d\|z\|^{2} \geqslant 1$.

Let $e=(1, \underbrace{0, \ldots, 0,0, \ldots, 0}_{N+M-1})^{t} \in \mathbb{C}^{N+M}$, then $\|z\|^{2}=0,\|w\|^{2}=1$.

Since $c\|w\|^{2}+d\|z\|^{2} \geqslant 1$, we get $c \geqslant 1$.

Let $e^{\prime}=(\underbrace{0, \ldots, 0}_{N}, 1, \underbrace{0, \ldots, 0}_{M-1})^{t} \in \mathbb{C}^{N+M}$, then $\|z\|^{2}=1,\|w\|^{2}=0$.

Since $c\|w\|^{2}+d\|z\|^{2} \geqslant 1$, we get $d \geqslant 1$.

Furthermore $V(R(c, d))=c^{-N} d^{-M} \omega_{N+M} \leqslant V\left(B^{N+M}\right)=\omega_{N+M}$.

From the above discussion, we can obtain that $B^{N+M}$ is the inscribed ball of $Y_{I I}(N, p, K)$, and it is also the unique maximal inscribed ball.

\section{The minimal circumscribed Hermitian ellipsoid}

Lemma 12. If $Y_{I I}(N, p, K) \subset S(a, b)(a>0, b>0)$, then $0<a \leqslant 1,0<b \leqslant \frac{1}{p}$.

Proof. Let $\forall(w, Z) \in Y_{I I}(N, p, K)$, where $Z \in \Re_{I I}(p)$. As for $Z$, there exists a unitary matrix $U$, such that

$$
Z=U^{t} \tilde{\Lambda} U
$$

and

$$
Z \bar{Z}=U^{t} \widetilde{\Lambda}^{2} \bar{U}
$$

where $\widetilde{\Lambda}$ is the same as the equation $(\star)$. 
Because $I-Z \bar{Z}>0, U^{t}\left(I-\widetilde{\Lambda}^{2}\right) \bar{U}>0$, furthermore we can infer

$$
0 \leqslant \tilde{\lambda}_{p}^{2} \leqslant \tilde{\lambda}_{p-1}^{2} \leqslant \cdots \leqslant \tilde{\lambda}_{1}^{2}<1 .
$$

Thus

$$
\operatorname{tr}(Z \bar{Z})=\operatorname{tr}\left(U^{t} \widetilde{\Lambda}^{2} \bar{U}\right)=\tilde{\lambda}_{1}^{2}+\tilde{\lambda}_{2}^{2}+\cdots+\tilde{\lambda}_{p}^{2}<p
$$

and

$$
\|w\|^{2 K}<\operatorname{det}(I-Z \bar{Z})=\left(1-\tilde{\lambda}_{1}^{2}\right)\left(1-\tilde{\lambda}_{2}^{2}\right) \cdots\left(1-\tilde{\lambda}_{p}^{2}\right) \leqslant 1 .
$$

Since $(w, Z) \in Y_{I I}(N, p, K) \subset S(a, b)(a>0, b>0)$, it follows that

$$
a\|w\|^{2} \leqslant a\|w\|^{2}+b \operatorname{tr}(Z \bar{Z})<1, b \operatorname{tr}(Z \bar{Z}) \leqslant a\|w\|^{2}+b \operatorname{tr}(Z \bar{Z})<1,
$$

furthermore $0<a \leqslant 1,0<b \leqslant \frac{1}{p}$.

For $\forall(w, Z) \in \partial Y_{I I}(N, p, K)$, we can obtain the following results:

According to the proof of Lemma 12, for $\forall(w, Z) \in \partial Y_{I I}(N, p, K)$, we get

$$
\|w\|^{2 K}=\operatorname{det}(I-Z \bar{Z})=\left(1-\tilde{\lambda}_{1}^{2}\right)\left(1-\tilde{\lambda}_{2}^{2}\right) \cdots\left(1-\tilde{\lambda}_{p}^{2}\right) .
$$

Let $\lambda_{i}=\tilde{\lambda}_{i}^{2}, i=1, \ldots, p$, then $\|w\|^{2 K}=\left(1-\lambda_{1}\right) \cdots\left(1-\lambda_{p}\right)$.

Thus

$$
\begin{aligned}
a\|w\|^{2}+b \operatorname{tr}(Z \bar{Z})= & a\left[\left(1-\lambda_{1}\right)\left(1-\lambda_{2}\right) \cdots\left(1-\lambda_{p}\right)\right]^{\frac{1}{K}} \\
& +b\left(\lambda_{1}+\lambda_{2}+\cdots+\lambda_{p}\right) \\
\leqslant & a\left(1-\frac{1}{p} \sum_{l=1}^{p} \lambda_{l}\right)^{\frac{p}{K}}+b \sum_{l=1}^{p} \lambda_{l} \\
= & a(1-\lambda)^{\frac{p}{K}}+b p \lambda
\end{aligned}
$$

where $\lambda=\frac{1}{p} \sum_{l=1}^{p} \lambda_{l}, l=1, \ldots, p$.

Let $g_{(a, b)}(\lambda)=a(1-\lambda)^{\frac{p}{K}}+b p \lambda$, with which we will discuss the conditions that $Y_{I I}(N, p, K) \subset S(a, b)$ and there are tangent points between them.

Lemma 13. $Y_{I I}(N, p, K) \subset S(a, b)$, and there are tangent points between them if and only if $\max _{\lambda \in[0,1]} g_{(a, b)}(\lambda)=1$.

Proof. (sufficiency)

When $\forall(w, Z) \in \partial Y_{I I}(N, p, K)$, we know from the above discussion,

$$
a\|w\|^{2}+b \operatorname{tr}(Z \bar{Z}) \leqslant a(1-\lambda)^{\frac{p}{K}}+b p \lambda \leqslant \max _{\lambda \in[0,1]} g_{(a, b)}(\lambda)=1,
$$

thus $(w, Z) \in \overline{S(a, b)}$, furthermore $Y_{I I}(N, p, K) \subset S(a, b)$. 
Let $\lambda_{0} \in[0,1), g_{(a, b)}\left(\lambda_{0}\right)=\max _{\lambda \in[0,1]} g_{(a, b)}(\lambda)=1$, and $\tilde{\lambda}_{10}=\tilde{\lambda}_{20}=\cdots=\tilde{\lambda}_{p 0}=$ $\lambda_{0}^{\frac{1}{2}}$, then we construct

$$
Z_{0}=U^{t}\left(\begin{array}{cccc}
\tilde{\lambda}_{10} & 0 & \cdots & 0 \\
0 & \tilde{\lambda}_{20} & \cdots & 0 \\
\cdots & \cdots & \cdots & \cdots \\
0 & 0 & \cdots & \tilde{\lambda}_{p 0}
\end{array}\right) U
$$

where $U$ is a unitary matrix. Obviously $Z_{0}^{t}=Z_{0}, I-Z_{0} \bar{Z}_{0}>0$, i.e., $Z_{0} \in$ $\Re_{I I}(p)$.

Let $w_{0}=\left(\left(\operatorname{det}\left(I-Z_{0} \bar{Z}_{0}\right)\right) \frac{1}{2 K}, 0, \ldots, 0\right) \in \mathbb{C}^{N}$, then $\left\|w_{0}\right\|^{2 K}=\operatorname{det}\left(I-Z_{0} \bar{Z}_{0}\right)$, i.e., $\left(w_{0}, Z_{0}\right) \in \partial Y_{I I}(N, p, K)$.

Moreover $a\left\|w_{0}\right\|^{2}+b \operatorname{tr}\left(Z_{0} \bar{Z}_{0}\right)=a\left(1-\lambda_{0}\right)^{\frac{p}{K}}+b p \lambda_{0}=g_{(a, b)}\left(\lambda_{0}\right)=1$, then $\left(w_{0}, Z_{0}\right) \in \partial S(a, b)$.

Hence $Y_{I I}(N, p, K) \subset S(a, b)$, and there exist tangent points between them. (necessity)

(1) Assume $\max _{\lambda \in[0,1]} g_{(a, b)}(\lambda)>1$, then let $\lambda_{0} \in[0,1)$, such that $g_{(a, b)}\left(\lambda_{0}\right)=$ $\max _{\lambda \in[0,1]} g_{(a, b)}(\lambda)$.

Let $\left(w_{0}, Z_{0}\right)$ be the same as the form in the above proof, then $\left\|w_{0}\right\|^{2 K}=$ $\operatorname{det}\left(I-Z_{0} \bar{Z}_{0}\right)$, i.e., $\left(w_{0}, Z_{0}\right) \in \partial Y_{I I}(N, p, K)$, and $a\left\|w_{0}\right\|^{2}+b \operatorname{tr}\left(Z_{0} \bar{Z}_{0}\right)=$ $g_{(a, b)}\left(\lambda_{0}\right)>1$, it is in contradiction to $Y_{I I}(N, p, K) \subset S(a, b)$.

(2) Assume $\max _{\lambda \in[0,1]} g_{(a, b)}(\lambda)<1$, then let $(\tilde{w}, \tilde{Z}) \in \partial Y_{I I}(N, p, k) \cap \partial S(a, b)$, i.e. $\|\tilde{w}\|^{2 K}=\operatorname{det}(I-\tilde{Z} \overline{\tilde{Z}})$ and $a\|\tilde{w}\|^{2}+b \operatorname{tr}(\tilde{Z} \tilde{Z})=1$.

But $a\|\tilde{w}\|^{2}+b \operatorname{tr}(\tilde{Z} \tilde{Z}) \leqslant g_{(a, b)}(\lambda) \leqslant \max _{\lambda \in[0,1]} g_{(a, b)}(\lambda)<1$, it contradict $(\tilde{w}, \tilde{Z}) \in$ $\partial S(a, b)$.

Generalizing from the above proof, the lemma is true.

If $Y_{I I}(N, p, K) \subset S(a, b)$, and there are tangent points between them, then $S(a, b)$ may be called the circumscribed domain of $Y_{I I}(N, p, K)$.

Next we are going to discuss the accurate form of the minimal circumscribed Hermitian ellipsoid of $Y_{I I}(N, p, K)$ :

(I) $K=p$

Lemma 14. When $K=p, S(a, b)$ is the circumscribed domain of $Y_{I I}(N, p, K)$ if and only if $b=\frac{1}{p}$ and $0<a \leqslant 1$, or $a=1$ and $0<b \leqslant \frac{1}{p}$.

Proof. When $K=p, g_{(a, b)}(\lambda)=a(1-\lambda)+b p \lambda=a+(b p-a) \lambda \quad \lambda \in[0,1]$.

From Lemma 12 and Lemma 13, we know:

(1) When $0<a \leqslant b p$ and $\lambda=1, g_{(a, b)}(\lambda)$ attains the maximum:

$$
\max _{\lambda \in[0,1]} g_{(a, b)}(\lambda)=g_{(a, b)}(1)=b p=1,
$$

then $b=\frac{1}{p}, 0<a \leqslant 1$; 
(2) When $a \geqslant b p>0$ and $\lambda=0, g_{(a, b)}(\lambda)$ attains the maximum:

$$
\max _{\lambda \in[0,1]} g_{(a, b)}(\lambda)=g_{(a, b)}(1)=a=1,
$$

then $0<b \leqslant \frac{1}{p}, a=1$.

Theorem 15. When $K=p$, the minimal circumscribed Hermitian ellipsoid of $Y_{I I}(N, p, K)$ is

$$
\left\{(w, z) \in \mathbb{C}^{N+M}:\|w\|^{2}+\frac{1}{p}\|z\|^{2}<1\right\} .
$$

Proof. Because of Proposition 3, the volume of the ellipsoid

$$
S(a, b)=\left\{(w, z) \in \mathbb{C}^{N+M}: a\|w\|^{2}+b\|z\|^{2}<1\right\} \quad a>0, b>0
$$

is

Hence

$$
V(S(a, b))=a^{-N} b^{-M} \omega_{N+M}
$$

$$
\begin{aligned}
& \min _{b=\frac{1}{p}, 0<a \leqslant 1} V(S(a, b))=V\left(S\left(1, \frac{1}{p}\right)\right)=p^{M} \omega_{N+M} ; \\
& \min _{a=1,0<b \leqslant \frac{1}{p}} V(S(a, b))=V\left(S\left(1, \frac{1}{p}\right)\right)=p^{M} \omega_{N+M} .
\end{aligned}
$$

Thus when $K=p, S\left(1, \frac{1}{p}\right)$ is the minimal circumscribed Hermitian ellipsoid of $Y_{I I}(N, p, K)$.

(II) $K>p$

Lemma 16. When $K>p, S(a, b)$ is the circumscribed domain of $Y_{I I}(N, p, K)$ if and only if $b p+b(K-p)\left(\frac{a}{b K}\right)^{\frac{K}{K-p}}=1$ and $0<a \leqslant K b$, or $a=1$ and $1 \geqslant K b>0$.

Proof. When $K>p, g_{(a, b)}(\lambda)=a(1-\lambda)^{\frac{p}{K}}+b p \lambda$.

It is easy to check that $g_{(a, b)}(\lambda)(\lambda \in[0,1])$ is a continuous function, and when $\lambda \neq 1$, it is differentiable :

$$
g_{(a, b)}^{\prime}(\lambda)=-\frac{a p}{K}(1-\lambda)^{\frac{p-K}{K}}+b p \quad(\lambda \neq 1) .
$$

Let $g_{(a, b)}^{\prime}(\lambda)=0$, we get

$$
\lambda_{0}=1-\left(\frac{a}{b K}\right)^{\frac{K}{K-p}} .
$$

(1) When $a \geqslant b K>0, \quad \lambda_{0} \in(-\infty, 0]$.

Now $g_{(a, b)}^{\prime}(\lambda) \leqslant 0(\lambda \in[0,1))$, then $g_{(a, b)}(\lambda)$ is decreasing in $[0,1)$, and $\lambda=0$ is the maximum point.

$$
g_{(a, b)}(0)=a, \quad g_{(a, b)}(1)=b p .
$$

Because $a \geqslant b K$ and $K>p, a>b p$. 
So $\max _{\lambda \in[0,1]} g_{(a, b)}(\lambda)=a$.

(2) When $0<a \leqslant b K, \lambda_{0} \in[0,1)$.

If $\lambda \in\left[0, \lambda_{0}\right], g_{(a, b)}^{\prime}(\lambda) \geqslant 0$; if $\lambda \in\left[\lambda_{0}, 1\right), g_{(a, b)}^{\prime}(\lambda) \leqslant 0$; then $\lambda_{0}$ is the maximum point of $g_{(a, b)}(\lambda)(\lambda \in[0,1))$.

Now

$$
\begin{aligned}
g_{(a, b)}\left(\lambda_{0}\right) & =b p+b(K-p)\left(\frac{a}{K b}\right)^{\frac{K}{K-p}}, \\
g_{(a, b)}(1) & =b p .
\end{aligned}
$$

For $K>p$ and $b(K-p)\left(\frac{a}{b K}\right)^{\frac{K}{K-p}}>0, g_{(a, b)}\left(\lambda_{0}\right)>b p$.

So

$$
\begin{aligned}
\max _{\lambda \in[0,1]} g_{(a, b)}(\lambda) & =g_{(a, b)}\left(\lambda_{0}\right)=b p+\left(\frac{b K}{a}\right)^{\frac{p}{p-K}}\left(a-\frac{a p}{K}\right) \\
& =b p+b(K-p)\left(\frac{a}{b K}\right)^{\frac{K}{K-p}} .
\end{aligned}
$$

By Lemma 13, we know the lemma is true.

Theorem 17. When $K>p$, the minimal circumscribed Hermitian ellipsoid of $Y_{I I}(N, p, K)$ is

$\left\{(w, z) \in \mathbb{C}^{N+M}: \frac{(2 N)^{\frac{K-p}{K}}(2 N+K p+K)^{\frac{p}{K}}}{2 N+p^{2}+p}\|w\|^{2}+\frac{2 N+(p+1) K}{2 N K+(p+1) p K}\|z\|^{2}<1\right\}$.

Proof. Because the volume of the minimal circumscribed Hermitian ellipsoid of $Y_{I I}(N, p, K)$ is

$$
V(S(a, b))=a^{-N} b^{-M} \omega_{N+M},
$$

we need consider the minimum of the following function to obtain the minimal circumscribed Hermitian ellipsoid of $Y_{I I}(N, p, K)$ :

$$
T(a, b):=a^{-N} b^{-M} \text {. }
$$

(1) When $0<a \leqslant b K$, we consider the minimum of the function

$$
T(a, b):=a^{-N} b^{-M}
$$

with the constraint

$$
b p+b(K-p)\left(\frac{a}{b K}\right)^{\frac{K}{K-p}}=1 .
$$

From the constraint, we obtain

$$
a=K(1-b p)^{\frac{K-p}{K}}(K-p)^{\frac{p-K}{K}} b^{\frac{p}{K}} .
$$

Then

$$
\begin{aligned}
T(a, b) & =\left[K(1-b p)^{\frac{K-p}{K}}(K-p)^{\frac{p-K}{K}} b^{\frac{p}{K}}\right]^{-N} b^{-M} \\
& =K^{-N}(K-p)^{\frac{(K-p) N}{K}}(1-b p)^{\frac{(p-K) N}{K}} b^{\frac{-p(2 N+K p+K)}{2 K}}:=T(b) . \\
T^{\prime}(b)= & (-p) K^{-N}(K-p)^{\frac{(K-p) N}{K}}(1-b p)^{\frac{(p-K) N-K}{K}} b^{\frac{-p(2 N+p K+K)-2 K}{2 K}}
\end{aligned}
$$




$$
\begin{aligned}
& \times\left[\frac{(p-K) N b}{K}+(1-b p) \frac{2 N+p K+K}{2 K}\right] \\
= & \frac{1}{2} p K^{-N-1}(K-p)^{\frac{(K-p) N}{K}}(1-b p)^{\frac{(p-K) N-K}{K}} \\
& \times b^{\frac{-p(2 N+p K+K)-2 K}{2 K}}\left(2 N b K+b K p^{2}+b p K-2 N-p K-K\right) .
\end{aligned}
$$

Let $T^{\prime}(b)=0$, then we get

$$
b_{0}=\frac{2 N+(p+1) K}{\left(2 N+p^{2}+p\right) K} .
$$

When $0<b<b_{0}, T^{\prime}(b)<0, T(b)$ is decreasing, and when $b_{0}<b<+\infty$, $T^{\prime}(b)>0, T(b)$ is increasing, so $T(b)$ attains the minimum at $b_{0}$.

Let $b=b_{0}$ in (i), then we get

$$
a_{0}=\frac{(2 N)^{\frac{K-p}{K}}(2 N+K p+K)^{\frac{p}{K}}}{2 N+p^{2}+p} .
$$

So

$$
\begin{aligned}
\min _{\substack{0<a \leqslant b K \\
Y_{I I} \subset S(a, b)}} T(a, b)= & T\left(a_{0}, b_{0}\right) \\
= & {\left[\frac{(2 N)^{\frac{K-p}{K}}(2 N+p K+K)^{\frac{p}{K}}}{2 N+p^{2}+p}\right]^{-N} } \\
& \times\left[\frac{2 N+p K+K}{\left(2 N+p^{2}+p\right) K}\right]^{-M} \\
= & (2 N)^{\frac{N(p-K)}{K}} K^{M} \frac{\left(2 N+p^{2}+p\right)^{\frac{2 N+p(p+1)}{2}}}{(2 N+K p+K)^{\frac{2 p N+p K(p+1)}{2 K}}} .
\end{aligned}
$$

(2) When $a \geqslant b K>0$, we consider the minimum of the function

$$
T(a, b):=a^{-N} b^{-M}
$$

with the constraint $a=1$.

Because $a \geqslant b K>0,0<b \leqslant \frac{a}{K}=\frac{1}{K}$.

Now

$$
\min _{\substack{a \geqslant b K>0 \\ Y_{I I}(N, p, K) \subset S(a, b)}} T(a, b)=\min _{a=1,0<b \leqslant \frac{1}{K}} T(a, b)=T\left(1, \frac{1}{K}\right)=K^{M} .
$$

Compare the minimal value of $T(a, b)$ in (1) and (2):

About (1),

$$
\begin{aligned}
T\left(a_{0}, b_{0}\right) & =(2 N)^{\frac{N(p-K)}{K}} K^{M} \frac{[2 N+p(p+1)]^{N}}{[2 N+K(p+1)]^{\frac{p N}{K}}}\left[\frac{2 N+p(p+1)}{2 N+K(p+1)}\right]^{M} \\
& =K^{M}\left[\frac{\left(1+\frac{p(p+1)}{2 N}\right)^{\left.\frac{(2 N}{p(p+1)}+1\right)}}{\left(1+\frac{K(p+1)}{2 N}\right)^{\left(\frac{2 N}{K(p+1)}+1\right)}}\right]^{M} .
\end{aligned}
$$



$0)$.

Here need we discuss the monotonicity of function $f(x)=(1+x)^{\left(\frac{1}{x}+1\right)}(x>$

$$
f^{\prime}(x)=(1+x)^{\left(\frac{1}{x}+1\right)}\left(\frac{x-\log (1+x)}{x^{2}}\right) .
$$

Let

$$
g(x)=x-\log (1+x) \quad(x>0),
$$

then $g^{\prime}(x)=1-\frac{1}{x+1}>0$ when $x>0$. Since $g(0)=0$, it follows that $g(x)>0(x>0)$, i.e., $x>\log (1+x)(x>0)$. So $f^{\prime}(x)>0(x>0)$, i.e., $f(x)$ is increasing strictly in $[0,+\infty)$.

With the above result, because $\frac{K(p+1)}{2 N}>\frac{p(p+1)}{2 N}$,

$$
\frac{\left(1+\frac{p(p+1)}{2 N}\right)^{\left(\frac{2 N}{p(p+1)}+1\right)}}{\left(1+\frac{K(p+1)}{2 N}\right)^{\left(\frac{2 N}{K(p+1)}+1\right)}}<1,
$$

furthermore

$$
T\left(a_{0}, b_{0}\right)<K^{M}
$$

Generalizing from the above proof, when $K>p, a=a_{0}$ and $b=b_{0}$, $T(a, b)$ attains the minimal value, and $V(S(a, b))$ attains the minimum, so $S\left(a_{0}, b_{0}\right)=\left\{(w, z) \in \mathbb{C}^{N+M}: a_{0}\|w\|^{2}+b_{0}\|z\|^{2}<1\right\}$ is the minimal circumscribed Hermitian ellipsoid of $Y_{I I}(N, p, K)$.

(III) $0<K<p$

Lemma 18. When $0<K<p, S(a, b)$ is the circumscribed domain of $Y_{I I}$ if and only if $b p=1$ and $0<a \leqslant b p$, or $a=1$ and $a \geqslant b p>0$.

Proof. When $0<K<p$,

$$
\begin{gathered}
g_{(a, b)}(\lambda)=a(1-\lambda)^{\frac{p}{K}}+b p \lambda, \\
g_{(a, b)}^{\prime}(\lambda)=-\frac{a p}{K}(1-\lambda)^{\frac{p-K}{K}}+b p \quad(\lambda \neq 1) .
\end{gathered}
$$

Let $g_{(a, b)}^{\prime}(\lambda)=0$, we get $\lambda_{0}=1-\left(\frac{b K}{a}\right)^{\frac{K}{p-K}}$.

(1) When $0<a \leqslant b K<b p, g_{(a, b)}^{\prime}(\lambda) \geqslant 0(\lambda \in[0,1))$, then $g_{(a, b)}(\lambda)$ is increasing in $[0,1)$, moreover when $\lambda \in[0,1), g_{(a, b)}(\lambda)<b p(1-\lambda)+b p \lambda \leqslant$ $b p=g_{(a, b)}(\mathbf{1})$, so

$$
\max _{\lambda \in[0,1]} g_{(a, b)}(\lambda)=g_{(a, b)}(1)=b p .
$$

(2) When $a \geqslant b K, 0 \leqslant \lambda_{0}<1$.

When $\lambda \in\left[0, \lambda_{0}\right], g_{(a, b)}^{\prime}(\lambda) \leqslant 0, g_{(a, b)}(\lambda)$ is decreasing, when $\lambda \in\left[\lambda_{0}, 1\right)$, $g_{(a, b)}^{\prime}(\lambda) \geqslant 0, g_{(a, b)}(\lambda)$ is increasing, then $g_{(a, b)}(\lambda)$ attains the minimum at $\lambda_{0}$. So

$$
\max _{\lambda \in[0,1], a \geqslant K b} g_{(a, b)}(\lambda)=\max \left\{g_{(a, b)}(0), g_{(a, b)}(1)\right\}=\max \{a, b p\}
$$


When $a \geqslant b p, \max _{\lambda \in[0,1]} g_{(a, b)}(\lambda)=g_{(a, b)}(0)=a$.

When $b K \leqslant a \leqslant b p, \max _{\lambda \in[0,1]} g_{(a, b)}(\lambda)=g_{(a, b)}(1)=b p$.

Generalizing from (1) and (2), when $0<K<p$, according to Lemma 13, $S(a, b)$ is the circumscribed domain of $Y_{I I}(N, p, K)$ if and only if $b p=1$ when $0<a \leqslant b p ; S(a, b)$ is the circumscribed domain of $Y_{I I}(N, p, K)$ if and only if $a=1$ when $a \geqslant b p>0$.

Theorem 19. When $0<K<p$, the minimal circumscribed Hermitian ellipsoid of $Y_{I I}(N, p, K)$ is

$$
\left\{(w, z) \in \mathbb{C}^{N+M}:\|w\|^{2}+\frac{1}{p}\|z\|^{2}<1\right\} .
$$

Proof. According to Proposition 3, the volume of $S(a, b)$ is

$$
V(S(a, b))=a^{-N} b^{-M} \omega_{N+M}
$$

Then

$$
\begin{aligned}
& \min _{b p=1,0<a \leqslant 1} V(S(a, b))=V\left(S\left(1, \frac{1}{p}\right)\right)=p^{M} \omega_{N+M} ; \\
& \min _{a=1,0<b \leqslant \frac{1}{p}} V(S(a, b))=V\left(S\left(1, \frac{1}{p}\right)\right)=p^{M} \omega_{N+M} .
\end{aligned}
$$

So when $a=1, b=\frac{1}{p}, S\left(1, \frac{1}{p}\right)=\left\{(w, z) \in \mathbb{C}^{N+M}:\|w\|^{2}+\frac{1}{p}\|z\|^{2}<1\right\}$ is the minimal circumscribed Hermitian ellipsoid of $Y_{I I}(N, p, K)$.

Generalizing from (I), (II), (III), when $0<K \leqslant p, S\left(1, \frac{1}{p}\right)=\{(w, z) \in$ $\left.\mathbb{C}^{N+M}:\|w\|^{2}+\frac{1}{p}\|z\|^{2}<1\right\}$ is the unique minimal circumscribed Hermitian ellipsoid of $Y_{I I}(N, p, K)$; when $K>p, S\left(a_{0}, b_{0}\right)=\left\{(w, z) \in \mathbb{C}^{N+M}: a_{0}\|w\|^{2}+\right.$ $\left.b_{0}\|z\|^{2}<1\right\}\left(a_{0}, b_{0}\right.$ here are the previous ones in Theorem 17) is the unique minimal circumscribed Hermitian ellipsoid of $Y_{I I}(N, p, K)$.

\section{Extremal mapping and extremal value of $Y_{I I}(N, p, K)$}

Theorem 20. When $0<K \leqslant p$, the $C$-extremal mapping from $Y_{I I}(N, p, K)$ to $B^{N+M}$ is:

$$
\begin{gathered}
f: Y_{I I}(N, p, K) \longrightarrow B^{N+M} \\
f_{i}((w, Z))=w_{i} \quad i=1,2, \ldots, N \\
f_{u v}((w, Z))=\left(\frac{1}{p}\right)^{\frac{1}{2}} z_{u v} \quad u=1,2, \ldots, p ; v=1,2, \ldots, p, u \leqslant v .
\end{gathered}
$$

Proof. Because $Y_{I I}(N, p, K)$ and $B^{N+M}$ are balanced holomorphic domains, by proposition $1, \forall l \in \operatorname{Hol}\left(Y_{I I}(N, p, K), B^{N+M}\right)$ satisfies

$$
d l(0)\left(Y_{I I}(N, p, K)\right) \subset B^{N+M} .
$$


Thus the extremal mapping $f$ from $Y_{I I}(N, p, K)$ to $B^{N+M}$ is a solution of the following extremal problem:

$\sup \left\{|\operatorname{det} d l(0)|: d l(0)\right.$ complex linear mapping, $\left.d l(0)\left(Y_{I I}(N, p, K)\right) \subset B^{N+M}\right\}$.

According to Theorem 19 , when $0<K \leqslant p$, the minimal circumscribed Hermitian ellipsoid of $Y_{I I}(N, p, K)$ is

$$
S\left(1, \frac{1}{p}\right)=\left\{(w, z) \in \mathbb{C}^{N+M}:\|w\|^{2}+\frac{1}{p}\|z\|^{2}<1\right\} .
$$

So by proposition $4, d f(0)\left(S\left(1, \frac{1}{p}\right)\right)=B^{N+M}$.

While

$$
B^{N+M}=\left\{(w, z) \in \mathbb{C}^{N+M}:\|w\|^{2}+\|z\|^{2}<1\right\}
$$

then the complex linear mapping from $S(a, b)$ to $B^{N+M}$ is

$$
\varphi=\left(\begin{array}{cc}
I^{(N)} & 0 \\
0 & \sqrt{\frac{1}{p}} I^{(M)}
\end{array}\right) .
$$

So $\varphi$ is just $d f(0)$, furthermore it can be inferred that the C-extremal mapping $f$ from $Y_{I I}(N, p, K)$ to $B^{N+M}$ is just the form in the theorem.

Theorem 21. When $K \geqslant 1$, the C-extremal mapping from $B^{N+M}$ to $Y_{I I}$ is:

$$
\begin{aligned}
g: B^{N+M} \longrightarrow Y_{I I}(N, p, K) \\
g(w, z)=(w, Z) .
\end{aligned}
$$

Proof. Because $Y_{I I}(N, p, K)$ and $B^{N+M}$ are balanced holomorphic domains, according to Proposition $1, \forall m \in \operatorname{Hol}\left(B^{N+M}, Y_{I I}(N, p, K)\right)$ satisfies

$$
d m(0)\left(B^{N+M}\right) \subset Y_{I I}(N, p, K)
$$

Thus the C-extremal mapping $g$ from $B^{N+M}$ to $Y_{I I}(N, p, K)$ is a solution of the following extremal problem:

$\sup \left\{|\operatorname{det} d m(0)|: d m(0)\right.$ complex linear mapping, $\left.d m(0)\left(B^{N+M}\right) \subset Y_{I I}\right\}$.

According to Proposition $4, d g(0)\left(B^{N+M}\right)$ is the maximal inscribed Hermitian ellipsoid of $Y_{I I}(N, p, K)$.

According to Proposition 11, when $K \geqslant 1, B^{N+M}$ is the maximal inscribed ellipsoid of $Y_{I I}(N, p, K)$.

Thus when $K \geqslant 1, d g(0)=I^{(N+M)}$, furthermore $g$ is just the form in the theorem.

Theorem 22. When $0<K \leqslant p$,

When $1 \leqslant K \leqslant p$,

$$
J_{\max }\left(Y_{I I}(N, p, K), B^{N+M}\right)=\left(\frac{1}{p}\right)^{\frac{p(p+1)}{4}} .
$$

$$
\mu\left(Y_{I I}(N, p, K), B^{N+M}\right)=\frac{p(p+1)}{4} \log p .
$$


Proof. When $0<K \leqslant p$, according to Theorem 20 and the definition of Cextremal value,

$$
J_{\max }\left(Y_{I I}(N, p, K), B^{N+M}\right)=|\operatorname{det} d f(0)|=\left(\frac{1}{p}\right)^{\frac{p(p+1)}{4}} .
$$

When $1 \leqslant K \leqslant p$, by Theorem 21 ,

$$
J_{\max }\left(B^{N+M}, Y_{I I}(N, p, K)\right)=|\operatorname{det} d g(0)|=1 .
$$

According to the definition of extremal distance,

$$
\begin{aligned}
\mu\left(Y_{I I}(N, p, K), B^{N+M}\right)= & -\log \left[J_{\max }\left(Y_{I I}(N, p, K), B^{N+M}\right)\right. \\
& \left.\cdot J_{\max }\left(B^{N+M}, Y_{I I}(N, p, K)\right)\right] \\
= & \frac{p(p+1)}{4} \log p .
\end{aligned}
$$

Theorem 23. When $K>p$, the $C$-extremal mapping from $Y_{I I}(N, p, K)$ to $B^{N+M} i s$ :

$$
\begin{gathered}
f: Y_{I I}(N, p, K) \rightarrow B^{N+M} \\
f_{i}((w, Z))=\sqrt{a_{0}} w_{i} \quad i=1,2, \ldots, N \\
f_{u v}((w, Z))=\sqrt{b_{0}} z_{u v} \quad u=1,2, \ldots, p ; v=1,2, \ldots, p, u \leqslant v,
\end{gathered}
$$

where $a_{0}, b_{0}$ are the previous ones in Theorem 17.

Proof. The method used to prove the theorem is similar to that of Theorem 20.

Now according to Theorem 17 ,

$$
d f(0)=\left(\begin{array}{cc}
\sqrt{a_{0}} I^{(N)} & 0 \\
0 & \sqrt{b_{0}} I^{(M)}
\end{array}\right) .
$$

In terms of the form of $d f(0)$, the C-extremal mapping $f$ from $Y_{I I}(N, p, K)$ to $B^{N+M}$ is just the one in the theorem.

Theorem 24. When $K>p$,

$$
\begin{gathered}
J_{\max }\left(Y_{I I}(N, p, K), B^{N+M}\right)=\frac{(2 N)^{\frac{N(K-p)}{2 K}}(2 N+p K+K)^{\frac{2 p N+K p(p+1)}{4 K}}}{K^{\frac{p(p+1)}{4}}\left(2 N+p^{2}+p\right)^{\frac{2 N+p(p+1)}{4}}}, \\
\mu\left(Y_{I I}(N, p, K), B^{N+M}\right)=\frac{1}{4 K} \log \frac{K^{K p(p+1)}\left(2 N+p^{2}+p\right)^{2 N K+K p(p+1)}}{(2 N)^{2 N(K-p)}(2 N+K p+K)^{2 p N+K p(p+1)}} .
\end{gathered}
$$

Proof. By Theorem 23 and the definition of C-extremal value,

$$
\begin{aligned}
J_{\max }\left(Y_{I I}(N, p, K), B^{N+M}\right) & =|\operatorname{det} d f(0)| \\
& =a_{0}^{\frac{N}{2}} b_{0}^{\frac{p(p+1)}{4}} \\
& =\frac{(2 N)^{\frac{N(K-p)}{2 K}}(2 N+p K+K)^{\frac{2 p N+K p(p+1)}{4 K}}}{K^{\frac{p(p+1)}{4}}\left(2 N+p^{2}+p\right)^{\frac{2 N+p(p+1)}{4}}} .
\end{aligned}
$$




$$
\begin{aligned}
\mu\left(Y_{I I}(N, p, K), B^{N+M}\right)= & -\log \left[J_{\max }\left(Y_{I I}(N, p, K), B^{N+M}\right)\right. \\
& \left.\cdot J_{\max }\left(B^{N+M}, Y_{I I}(N, p, K)\right)\right] \\
= & \frac{1}{4 K} \log \frac{K^{K p(p+1)}\left(2 N+p^{2}+p\right)^{2 N K+K p(p+1)}}{(2 N)^{2 N(K-p)}(2 N+K p+K)^{2 p N+K p(p+1)}} .
\end{aligned}
$$

Part IV. The extremal problems on $Y_{I}(N ; m, n ; K)$ and $Y_{I I I}(N, q, K)$

\section{Extremal mapping and extremal value of $Y_{I}(N ; m, n ; K)$}

Proposition $25([8])$. When $K \geqslant 1$, the unit hyperball $B^{N+m n}$ is the maximal inscribed ball of $Y_{I}(N ; m, n ; K)$.

Theorem 26 ([8]). When $0<K \leqslant m$, the minimal circumscribed Hermitian ellipsoid of $Y_{I}(N ; n, m ; K)$ is

$$
\left\{(w, z) \in \mathbb{C}^{N+m n}:\|w\|^{2}+\frac{1}{m}\|z\|^{2}<1\right\} .
$$

Theorem $27([8])$. When $K>m$, the minimal circumscribed Hermitian ellipsoid of $Y_{I}(N ; m, n ; K)$ is

$$
\left\{(w, z) \in \mathbb{C}^{N+m n}: \frac{N^{1-\frac{m}{K}}(N+n K)^{\frac{m}{K}}}{N+m n}\|w\|^{2}+\frac{N+n K}{K(N+m n)}\|z\|^{2}<1\right\} .
$$

Theorem 28 ([8]). When $0<K \leqslant m$, the C-extremal mapping from

$$
Y_{I}(N ; m, n ; K)
$$

to $B^{N+m n}$ is:

$$
\begin{gathered}
f: Y_{I}(N ; m, n ; K) \longrightarrow B^{N+m n} \\
f_{i}((w, Z))=w_{i} \quad i=1,2, \ldots, N \\
f_{u v}((w, Z))=\left(\frac{1}{m}\right)^{\frac{1}{2}} z_{u v} \quad u=1,2, \ldots, m ; v=1,2, \ldots, n .
\end{gathered}
$$

Theorem 29 ([8]). When $K \geqslant 1$, the C-extremal mapping from $B^{N+m n}$ to $Y_{I}(N ; m, n ; K)$ is:

$$
\begin{gathered}
g: B^{N+m n} \longrightarrow Y_{I}(N ; m, n ; K) \\
g(w, z)=(w, Z) .
\end{gathered}
$$

Theorem 30 ([8]). When $0<K \leqslant m$,

$$
J_{\max }\left(Y_{I}(N ; m, n ; K), B^{N+m n}\right)=m^{-\frac{m n}{2}} .
$$

When $1 \leqslant K \leqslant m$,

$$
\mu\left(Y_{I}(N ; m, n ; K), B^{N+m n}\right)=\frac{m n}{2} \log m .
$$


Theorem 31 ([8]). When $K>m$, the $C$-extremal mapping from

$$
Y_{I}(N ; m, n ; K)
$$

to $B^{N+m n}$ is.

$$
\begin{aligned}
f: Y_{I}(N ; m, n ; K) \longrightarrow B^{N+m n} \\
f_{i}((w, Z))=\sqrt{a_{0}} w_{i} \quad i=1,2, \ldots, N \\
f_{u v}((w, Z))=\sqrt{b_{0}} z_{u v} \quad u=1,2, \ldots, m ; v=1,2, \ldots, n,
\end{aligned}
$$

where $a_{0}=\frac{N^{1-\frac{m}{K}}(N+n K)^{\frac{m}{K}}}{N+m n}, b_{0}=\frac{N+n K}{K(N+m n)}$.

Theorem 32 ([8]). When $K>m$,

$$
\begin{gathered}
J_{\max }\left(Y_{I}(N ; m, n ; K), B^{N+m n}\right)=\frac{N^{\frac{N}{2}\left(1-\frac{m}{K}\right)}(N+n K)^{\frac{m}{2}\left(n+\frac{N}{K}\right)}}{K^{\frac{m n}{2}}(N+m n)^{\frac{N+m n}{2}}}, \\
\mu\left(Y_{I}(N ; m, n, K), B^{N+m n}\right)=\frac{1}{2} \log \frac{K^{m n}(N+m n)^{N+m n}}{N^{N\left(1-\frac{m}{K}\right)}(N+n K)^{m\left(n+\frac{N}{K}\right)}} .
\end{gathered}
$$

The above conclusion about the C-extremal problems on $Y_{I}(N ; m, n ; K)$ was gotten by $\mathrm{Su}$ jianbing, and the proof can be seen in the reference [8].

\section{Extremal mapping and extremal value of $Y_{I I I}(N, q, K)$}

Proposition 33. When $K \geqslant 2$, the unit hyperball $B^{N+\frac{q(q-1)}{2}}$ is the maximal inscribed ball of $Y_{I I I}(N, q, K)$.

Theorem 34. When $0<K \leqslant 2\left[\frac{g}{2}\right]$, the minimal circumscribed Hermitian ellipsoid of $Y_{I I I}(N, q, K)$ is

$$
\left\{(w, z) \in \mathbb{C}^{N+\frac{q(q-1)}{2}}:\|w\|^{2}+\frac{1}{\left[\frac{q}{2}\right]}\|z\|^{2}<1\right\} .
$$

Theorem 35. When $K>2\left[\frac{q}{2}\right]$, the minimal circumscribed Hermitian ellipsoid of $Y_{I I I}(N, q, K)$ is

$$
\begin{gathered}
\left\{(w, z) \in \mathbb{C}^{N+\frac{q(q-1)}{2}}: \frac{\left(4\left[\frac{q}{2}\right] N\right)^{\frac{K-2\left[\frac{q}{2}\right]}{K}}\left[4\left[\frac{q}{2}\right] N+K q(q-1)\right]^{\frac{2\left[\frac{q}{2}\right]}{K}}}{2\left[\frac{q}{2}\right](2 N+q(q-1))}\|w\|^{2}\right. \\
\left.+\frac{4\left[\frac{q}{2}\right] N+K q(q-1)}{\left[\frac{q}{2}\right](2 K N+K q(q-1))}\|z\|^{2}<1\right\} .
\end{gathered}
$$

Theorem 36. When $K \geqslant 2$, the C-extremal mapping from $B^{N+\frac{q(q-1)}{2}}$ to $Y_{I I I}(N, q, K)$ is:

$$
\begin{gathered}
g: \quad B^{N+\frac{q(q-1)}{2}} \longrightarrow Y_{I I I}(N, q, K) \\
g(w, z)=(w, z) I^{\left(N+\frac{q(q-1)}{2}\right)} .
\end{gathered}
$$


Theorem 37. When $0<K \leqslant 2\left[\frac{q}{2}\right]$, the C-extremal mapping from

$$
Y_{I I I}(N, q, K)
$$

to $B^{N+\frac{q(q-1)}{2}}$ is:

$$
\begin{aligned}
h: Y_{I I I}(N, q, K) & \longrightarrow B^{N+\frac{q(q-1)}{2}} \\
h(w, Z) & =(w, Z) H
\end{aligned}
$$

where

$$
H=\left(\begin{array}{cc}
I^{(N)} & 0 \\
0 & \sqrt{\frac{1}{\left[\frac{q}{2}\right]}}^{\left(\frac{q(q-1)}{2}\right)}
\end{array}\right)
$$

Theorem 38. When $0<K \leqslant 2\left[\frac{q}{2}\right]$,

$$
J_{\max }\left(Y_{I I I}(N, q, K), B^{N+\frac{q(q-1)}{2}}\right)=\left(\frac{1}{\left[\frac{q}{2}\right]}\right)^{\frac{q(q-1)}{4}} .
$$

When $1 \leqslant K \leqslant 2\left[\frac{q}{2}\right]$,

$$
\mu\left(Y_{I I I}(N, q, K), B^{N+\frac{q(q-1)}{2}}\right)=\frac{q(q-1)}{4} \log \left[\frac{q}{2}\right] .
$$

Theorem 39. When $K>2\left[\frac{q}{2}\right]$, the C-extremal mapping from $Y_{I I I}(N, q, K)$ to $B^{N+\frac{q(q-1)}{2}}$ is:

$$
\begin{aligned}
h: Y_{I I I}(N, q, K) & \longrightarrow B^{N+\frac{q(q-1)}{2}} \\
h(w, Z) & =(w, Z) H
\end{aligned}
$$

where

$$
H=\left(\begin{array}{cc}
\sqrt{a_{0}} I^{(N)} & 0 \\
0 & \sqrt{b_{0}} I^{\left(\frac{q(q-1)}{2}\right)}
\end{array}\right),
$$

and

$$
\begin{gathered}
a_{0}=\frac{\left(4\left[\frac{q}{2}\right] N\right)^{\frac{K-2\left[\frac{q}{2}\right]}{K}}\left[4\left[\frac{q}{2}\right] N+K q(q-1)\right]^{\frac{2\left[\frac{q}{2}\right]}{K}}}{2\left[\frac{q}{2}\right](2 N+q(q-1))}, \\
b_{0}=\frac{4\left[\frac{q}{2}\right] N+K q(q-1)}{\left[\frac{q}{2}\right](2 K N+K q(q-1))} .
\end{gathered}
$$

Theorem 40. When $K>2\left[\frac{q}{2}\right]$,

$$
\begin{gathered}
J_{\max }\left(Y_{I I I}(N, q, K), B^{N+\frac{q(q-1)}{2}}\right) \\
=\frac{(2 N)^{\frac{N\left(K-2\left[\frac{q}{2}\right]\right)}{2 K}}\left(2 N+\frac{k q(q-1)}{2\left[\frac{q}{2}\right]}\right)^{\frac{4\left[\frac{q}{2}\right] N+K q(q-1)}{4 K}} 2^{\frac{q(q-1)}{4}}}{K^{\frac{q(q-1)}{4}}(2 N+q(q-1))^{\frac{2 N+q(q-1)}{4}}}, \\
\mu\left(Y_{I I I}(N, q, K), B^{N+\frac{q(q-1)}{2}}\right) \\
=\frac{1}{4 K} \log \frac{K^{K q(q-1)}(2 N+q(q-1))^{2 N K+K q(q-1)}}{(2 N)^{2 N\left(K-2\left[\frac{q}{2}\right]\right)}\left(2 N+\frac{K q(q-1)}{2\left[\frac{q}{2}\right]}\right)^{4\left[\frac{q}{2}\right] N+K q(q-1)} 2^{K q(q-1)}} .
\end{gathered}
$$


To sum up, we discuss the C-extremal problems on

$$
Y_{I}(N ; m, n ; K), Y_{I I}(N, p, K)
$$

and $Y_{I I I}(N, q, K)$ in this paper. Because the method to deal with the Cextremal problem on $Y_{I V}(N ; n ; K)$ is different from the above discussion, the conclusions about the C-extremal mappings between $Y_{I V}(N ; n ; K)$ and $B^{n}$ will be given in the succeeding paper.

\section{References}

[1] C. Carathéodory, Über die Abbildungen, die durch Systeme von analytischen Funktionen von mehreren Veranderlichen erzeugt werden, Math. Z. 34 (1932), no. 1, 758-792.

[2] M. Daowei, Boundary behavior of invariant metrics and volume forms on strongly pseudoconvex domains, Duke Math. J. 63 (1991), no. 3, 673-697.

[3] _ Carathéodory extremal maps of ellipsoids, J. Math. Soc. Japan 49 (1997), no. 4, 723-739.

[4] Y. Kubota, An extremal problem on the classical Cartan domains. III, Kodai Math. J. 5 (1982), no. 3, 402-407.

[5] —_ An extremal problem on bounded symmetric domains, Bull. London Math. Soc. 15 (1983), no. 2, 126-130.

[6] L. Qikeng, The classical manifolds and classical domains, Shanghai Scientific Technical Publishers, 1963.

[7] Y. Weiping, The Bergman kernels on Cartan-Hartogs domains, Chinese Sci. Bull. 44 (1999), no. 21, 1947-1951.

[8] Y. Weiping and S. Jianbing, Extremal problems on super-Cartan domain of the first type, Complex Var. Theory Appl. 48 (2003), no. 5, 441-452.

AN WANG

DEPARTMENT OF MATHEMATICS

Capital normal University

BEIJing 100037, China

E-mail address: wangan@mail.cnu.edu.cn

XIN ZHAO

DEPaRTMENT OF Mathematics

Capital Normal University

BEIJING 100037, China

E-mail address: lettertozhaoxin@163.com

ZHIYIN LiU

Department of Mathematics

Capital Normal University

Beijing 100037, China

E-mail address: yuekf@sina.com 